\title{
162 Spread of infection in suppurative sinusitis
}

A Osteitis occurs in compact bone.

B Osteomyelitis occurs in diploic bone.

C Meningitis usually arises via a septic thrombophlebitis.

D Lymphatic channels are involved in the formation of subperiosteal abscesses.

E Spread into the subarachnoid space can occur via the perineural space around the olfactory nerves.

\section{Osteomyelitis of the frontal bone}

A Is most frequently due to frontal sinusitis.

B Streptococci and staphylococci are the commonest bacteria responsible.

C The onset is usually acute with rigors or epileptic fits.

D Pott's puffy tumour is a malignant myxomatous degeneration.

E Intracranial complications are a remote possibility.

\section{Osteomyelitis of the frontal bone}

A X-rays do not show any bony abnormality until sequestration occurs.

B Antibiotic treatment is ineffective.

C The floor of the frontal sinus should be trephined in severe cases with sequestration and intracranial infection.

D Opening the anterior wall of the sinus is contraindicated.

E Where bone has to be removed in young patients, the defect may regenerate spontaneously.

\section{Orbital complications of suppurative sinusitis}

A All the paranasal sinuses border the orbit at some point.

B Orbital complications are most common in children.

C A subperiosteal abscess due to frontal sinusitis points anteriorly.

D Orbital cellulitis usually precedes abscess formation.

E Thrombophlebitis may result in cavernous sinus thrombosis. 